\title{
Usefulness of waveform analysis of popliteal artery in Type II diabetic patients using gated magnetic resonance 2D-cine-PC imaging and ${ }^{31} \mathrm{P}$ spectroscopy
}

\author{
E.Suzuki ${ }^{1}$, A. Kashiwagi ${ }^{1}$, Y. Nishio ${ }^{1}$, H.Kojima ${ }^{1}$, H. Maegawa ${ }^{1}$, M. Haneda ${ }^{1}$, H. Yasuda ${ }^{1}$, S. Morikawa ${ }^{2}$, T. Inubushi ${ }^{2}$, \\ R.Kikkawa ${ }^{1}$ \\ ${ }^{1}$ Third Department of Medicine, Shiga University of Medical Science, Shiga, Japan \\ ${ }^{2}$ Molecular Neurobiology Research Centre, Shiga University of Medical Science, Shiga, Japan
}

\begin{abstract}
Aims/hypothesis. We studied 76 patients with Type II (non-insulin-dependent) diabetes mellitus and 16 age-matched non-diabetic subjects (control group) to clarify qualitative and quantitative abnormalities of waveform and flow volume of the popliteal artery. Methods. The 76 diabetic patients comprised 16 patients with occlusive arterial disease in the lower extremities [arteriosclerosis obliterans (ASO) group] and 60 patients free from this disease (non-ASO group). We flow analysed the popliteal artery and measured the phosphocreatine to inorganic phosphate ratio of resting plantar muscles to identify risk factors for foot lesions using gated magnetic resonance two-dimensional cine-mode phase-contrast imaging and ${ }^{31} \mathrm{P}$ spectroscopy.

Results. The control and non-ASO groups had a triphasic waveform with systolic, early and late diastolic components. All ASO patients had an abnormal monophasic waveform and a lower ankle brachial index than that of the control and non-ASO groups.
\end{abstract}

To clarify the mechanism of reduced flow volume of lower extremities, we assigned the 60 patients of the non-ASO group to the three subgroups based on their levels of total flow volume of the popliteal artery. The lowest group showed an abnormal triphasic waveform with lower amplitudes of systolic and late diastolic components and flow velocities in foot arteries than those of the highest group although ABI was similar. From stepwise multiple regression analysis, late diastolic flow volume was identified as an independent determinant for the phosphocreatine to inorganic phosphate ratio $\left(r^{2}=0.484, p<0.001\right)$.

Conclusion/interpretation. Waveform analysis of popliteal artery provides a powerful tool for identifying impaired peripheral circulation caused by either occlusive arterial disease or increased arterial resistance in diabetic patients. [Diabetologia (2000) 43:1031-1038]

Keywords Magnetic resonance, popliteal artery, waveform, flow volume, occlusive arterial disease, arterial resistance, plantar muscle, high energy phosphate content.
Received: 24 January 2000 and in revised form: 31 March 2000

Corresponding author: A. Kashiwagi MD, Third Department of Medicine, Shiga University of Medical Science, Seta, Tsukinowa-cho, Otsu, Shiga, 520-2192, Japan

Abbreviations: 2D-cine-PC: Two-dimensional cine-mode phase-contrast, $\mathrm{PCr}$ : phosphocreatine, $\mathrm{P}_{\mathrm{i}}$ : inorganic phosphate, ABI: ankle brachial index, $\mathrm{TcPO}_{2}$ : transcutaneous $\mathrm{O}_{2}$ tension, $\mathrm{MCV}$ : motor nerve conduction velocity, SCV: sensory nerve conduction velocity, $\mathrm{CV}_{\mathrm{R}-\mathrm{R}}$ : coefficient variation of the $\mathrm{R}-\mathrm{R}$ interval, FPG: fasting plasma glucose, sBP: systolic blood pressure, dBP: diastolic blood pressure, ASO: arteriosclerosis obliterans.
The ischaemic condition, neurological disorders [1] and abnormal mechanical forces [2] on the sole are predisposing factors for the development of diabetic foot lesions. The ischaemic condition, which results from low perfusion pressure or high arterial resistance with inadequate blood supply to the lower limbs, leads to subcutaneous and muscle tissue damage in the foot. Thus, assessments of the peripheral circulation in the lower limbs are beneficial for identifying high-risk patients prone to develop foot lesions.

Sonographic evaluation [3], measurement of ankle brachial index [4], plethysmography, transcutaneous 
$\mathrm{O}_{2}$ tension on the dorsal foot [5] and thermography are generally used to estimate haemodynamic changes in the peripheral circulation of the lower limbs. These methods have, however, limitations arising from various systemic and local factors, such as compact bone structure of the foot, calcification of the vessel wall, thickness of the skin and the presence of inflammation or local oedema. These conditions are particularly frequent in diabetic patients with foot lesions. Contrast angiography has been used to evaluate morphological changes in peripheral arteries but is frequently limited due to the nephrotoxity of the contrast medium in diabetic patients with complications. Magnetic resonance angiography with no contrast medium provides an excellent resolution of deep vasculatures through thick body tissues and morphological information of individual vessels covering a wide field of view. We recently reported that ${ }^{1} \mathrm{H}$ - and ${ }^{31} \mathrm{P}$-magnetic resonance spectroscopy and imaging were valuable as a new diagnostic tool for evaluating neuropathic foot ulcers in diabetic patients [6]. Furthermore, gated magnetic resonance two-dimensional cine-mode phase-contrast (2D-cine-PC) imaging can provide physiological flow data such as direction, velocity and volume [7].

The aim of this study was to define the abnormal peripheral circulation caused by either occlusive arterial diseases or increased arterial resistance in the lower extremities using gated magnetic resonance 2D-cine-PC imaging and flow analysis of the popliteal artery. Furthermore, these findings were analysed for energy metabolism of plantar muscles assessed by ${ }^{31} \mathrm{P}$-magnetic resonance spectroscopy to help identify high-risk patients for foot lesions.

\section{Subjects and methods}

Diabetic patients and control subjects. We admitted 76 patients with Type II (non-insulin-dependent) diabetes mellitus ranging in age from 50 to 69 years and 16 age-matched non-diabetic subjects (control group) consecutively to our hospital between July 1997 and February 2000. All 76 diabetic patients were admitted for strict glycaemic control or assessment of diabetic complications and 16 of these patients had occlusive arterial diseases in the lower extremities [arteriosclerosis obliterans (ASO) group]. The other 60 patients were free from these diseases (non-ASO group). The control group was diagnosed according to the World Health Organisation criteria [8] and had no history of hypertension or dyslipidaemia. In the ASO group, the presence of occlusive lesions and developing collateral arterial branches from the aortic bifurcation to the femoral artery were confirmed by intravenous digital subtraction angiography. These patients suffered from intermittent claudication but no resting pain or ischaemic foot ulcers. In the nonASO group, the ankle-brachial systolic blood pressure index (ABI) was greater than 0.9 [4] and intermittent claudication, resting pain and ischaemic foot ulcers were not complicated. These patients were fully mobile and were neither athletic nor sedentary with disuse atrophy of foot muscles following immobilisation. Patients who had heart failure, liver cirrhosis, severe nephropathy (serum creatinine greater than $133 \mu \mathrm{mol} / \mathrm{l}$ or with foot oedema), alcohol abuse, non-diabetic neurological disorders, myopathies and acute illness were excluded from the study. The study was approved by the ethics committee of our institution and informed consent was obtained from all patients before the examinations, which were done during their stay in hospital.

Clinical evaluations. Fasting plasma glucose (FPG) and glycated haemoglobin $\left(\mathrm{HbA}_{1 \mathrm{c}}\right)$ were examined to assess glycaemic control. Neurological assessments were done by motor nerve conduction velocity (MCV) of the posterior tibial nerve and sensory nerve conduction velocity (SCV) of the sural nerve using electromyography (Medelec MS-25, San-ei, Tokyo, Japan). Autonomic function was evaluated from the coefficient variation of the R-R interval $\left(\mathrm{CV}_{\mathrm{R}-\mathrm{R}}\right)$ during deep breathing monitored on an electrocardiogram (Cardimax FX-3301, Fukuda Denshi, Kyoto, Japan). The ABI was examined using a handheld ultrasound Doppler (ES-1000SP, Nihon Kohden, Tokyo, Japan) to assess occlusive arterial diseases in the lower extremities [4]. Transcutaneous $\mathrm{O}_{2}$ tension $\left(\mathrm{TcPO}_{2}\right)$ on the dorsal aspect of the first intermetatarsal space of patients at rest in the supine position was measured at $44^{\circ} \mathrm{C}$ using a cutaneous $\mathrm{PO}_{2}$ monitor (Cutaneous $\mathrm{PO}_{2}$ Monitor 8000, Kohken Medical, Tokyo, Japan) to assess the cutaneous perfusion [5]. A trained ophthalmologist carried out fundus ophthalmoscopies and defined diabetic patients as either without retinopathy, having simple retinopathy corresponding to levels $21-53$ or proliferative retinopathy to levels $60-80$ of the modified Airlie House System [9]. Furthermore, diabetic patients with normoalbuminuria, microalbuminuria and overt proteinuria were defined as having a urinary albumin excretion rate of less than 15 , 15-199 and greater than $200 \mu \mathrm{g} / \mathrm{min}$ by a 24 -h urine collection in our university hospital. These patients were also defined by their smoking habits as being a current smoker or non-smoker.

Magnetic resonance equipment. A magnetic resonance imaging scanner at $1.5 \mathrm{~T}$ (Signa Horizon-LX, GE Medical Systems, Milwaukee, Wis., USA) was used for the experimental protocols to assess the circulation of the popliteal artery and energy metabolism of plantar muscles. All patients were at rest in the supine position during examinations, which were done in a temperature-controlled room at $25^{\circ} \mathrm{C}$.

Flow analysis of popliteal artery and vasculature of foot arteries. Flow analysis of the popliteal artery and evaluation of morphological findings in the foot arteries without contrast medium were done using a quadrature head coil. To set up the individual flow analysis, the popliteal artery was depicted by twodimensional time-of-flight angiography with $28 \mathrm{~cm}$ field of view, $2 \mathrm{~mm}$ scan thickness, $256 \times 192$ acquisition matrix and 2 excitations. A single slice was oriented perpendicularly to the flow direction and flow data were obtained using two-dimensional cine-mode phase-contrast (2D-cine-PC) imaging triggered by peripheral gating, $80 \mathrm{~cm} / \mathrm{s}$ velocity encoding, $20 \mathrm{~cm}$ field of view, $5 \mathrm{~mm}$ scan thickness, $256 \times 256$ acquisition matrix and 2 excitations [7]. Heart rate was monitored by peripheral gating. Flow data were analysed with the flow analysis version 5.8 software package (GE Medical Systems, Milwaukee, Wis., USA) to determine direction, velocity and volume. Instantaneous flow volume at 16 equally spaced time points through the cardiac cycle was calculated from the individual velocity images by integrating the velocity across the area of the vessel. The resultant 16 velocities or flow volumes allowed assessment of flow variations in pulsatility and haemodynamics during the cardiac cycle. The control group had a typically triphasic waveform of the popliteal artery which could clearly be separated 

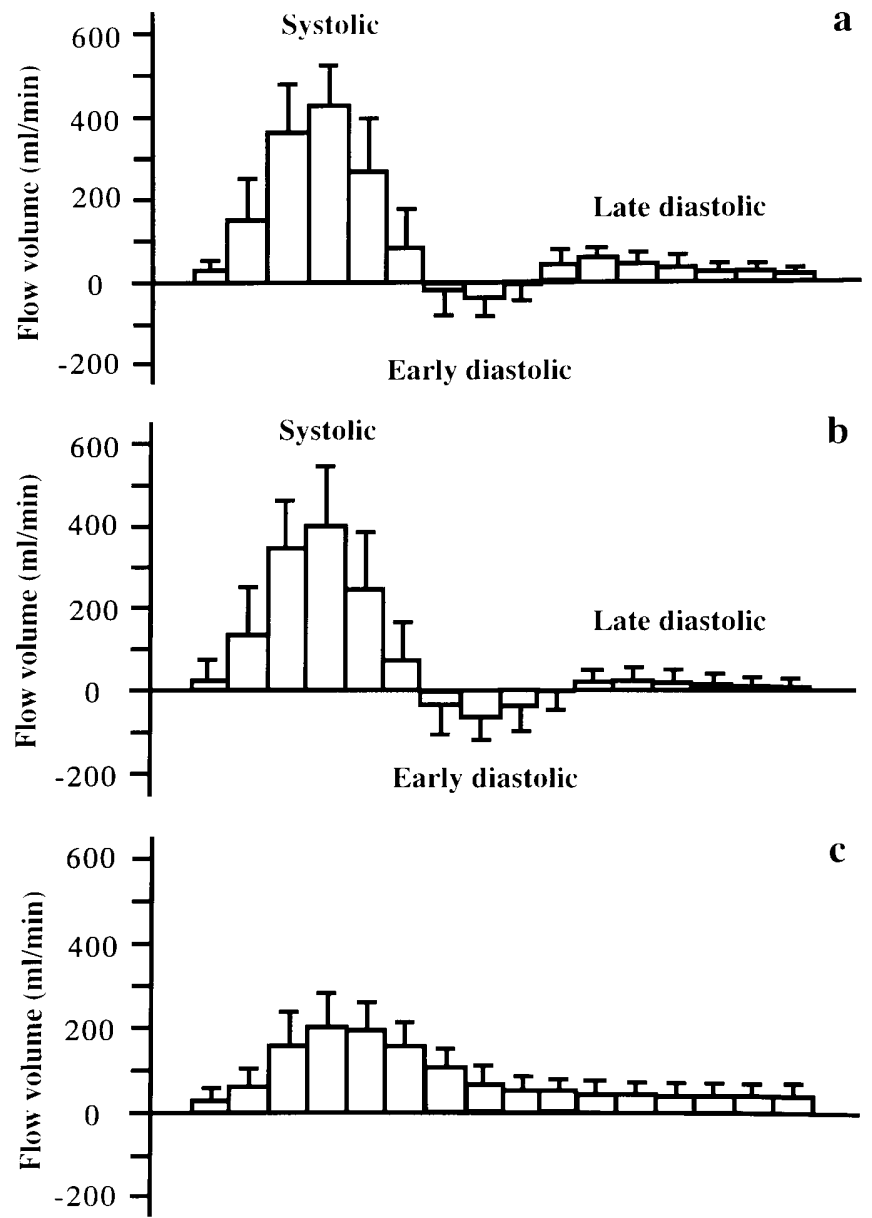

Fig. 1a-c. Waveform analyses of the popliteal artery in 16 diabetic patients with occlusive arterial diseases in the lower extremities $\mathbf{c}$ and 60 patients free from this disease $\mathbf{b}$ and 16 age-matched non-diabetic subjects a. Instantaneous flow volume at 16 equally spaced time points through the cardiac cycle were reconstructed. Data are expressed as means \pm SD

into systolic, early and late diastolic phases (Fig.1). Flow volumes of systolic, early diastolic and late diastolic phases of the cardiac cycle were calculated from the integration of the waveform. A resistive index, which allows quantitative analysis of the waveform and associates with arterial resistance, was defined as (A-B)/A, where $\mathrm{A}$ is the systolic peak velocity and $\mathrm{B}$ is the end-diastolic velocity [10].

To assess the morphological findings of foot arteries with no contrast medium, three-dimensional phase-contrast angiography was done in 60 slices with $2 \mathrm{~mm}$ scan thickness, $24 \mathrm{~cm}$ field of view and $5 \mathrm{~cm} / \mathrm{s}$ velocity encoding.

Energy metabolism of plantar muscles. An $8 \mathrm{~cm}$ diameter surface coil tuned to the phosphorus-31 frequency (25.9 MHz) was set at the plantar aspect of the first metatarsal head in the patients. A slice of $25 \mathrm{~mm}$ thickness in the plantar muscles parallel to the plane of the surface coil was selected with depth-resolved surface-coil spectroscopy [11]. Measurements of phosphorus compounds were obtained using ${ }^{31} \mathrm{P}$-magnetic resonance spectroscopy with a $1.5 \mathrm{~s}$ pulse repetition time and 512 excitations. The relative value of the phosphocreatine to inorganic phosphate $\left(\mathrm{PCr}: \mathrm{P}_{\mathrm{i}}\right)$ ratio was calculated from each peak area to help identify high-risk patients for foot lesions [12].
Statistical analysis. Statistical evaluation was done using StatView-J 5.0 software (SAS Institute, Cary, N.C., USA, 1992 and 1998) on a Macintosh computer. A multiple comparison of significant differences among the three groups was analysed by one-way analysis of variance (one-way ANOVA) followed by Scheffe's $F$ test. The chi-squared test for 2 by 2 or Bonferroni test for 2 by 3 contingency table was used to compare the frequencies between two or among three groups. Stepwise multiple regression analysis was done to evaluate the associations among the PCr: $\mathrm{P}_{\mathrm{i}}$ ratio of plantar muscles and various clinical characteristics of the subjects. Diabetic retinopathy and nephropathy were classified into three groups based on the severity. The $F$ value used was set at 4.0 at each step. We considered $p$ values less than 0.05 to be statistically significant. Data were expressed as means \pm SD.

\section{Results}

The characteristics of diabetic patients who had occlusive arterial diseases in the lower extremities (ASO group) and those free from these diseases (non-ASO group) and age-matched non-diabetic subjects (control group) are shown in Table 1. There were significant differences in $\mathrm{MCV}, \mathrm{SCV}$ and $\mathrm{CV}_{\mathrm{R}-\mathrm{R}}$ among the groups with the exception of cholesterol, HDL-cholesterol, triglycerides, systolic blood pressure (sBP) and diastolic blood pressure (dBP). In the non-ASO and ASO groups MCV, SCV and $\mathrm{CV}_{\mathrm{R}-\mathrm{R}}$ were lower than those of the control group. In the ASO group, the frequency of smokers was higher than that in the non-ASO group. Waveforms of the popliteal artery in the entire cardiac cycle are shown in Figure 1. Instantaneous flow volumes at 16 equally spaced time points through the cardiac cycle were reconstructed. The control group had a typically triphasic waveform of the popliteal artery, which could be clearly separated into systolic, early and late diastolic phases of the cardiac cycle (Fig.1). The affected extremities in the ASO group had an abnormal monophasic waveform which consisted of a reducing amplitude and broadening forward flow with the replacement of early diastolic flow reversal by persistent forward flow during the cardiac cycle, whereas the non-ASO group had a triphasic waveform (Fig.1). The quantitative analyses of peripheral circulation in these groups are shown in Table 2. There were significant differences in $\mathrm{ABI}, \mathrm{TcPO}_{2}$ and resistive index among the groups with the exception of total flow volume. In the non-ASO group, $\mathrm{TcPO}_{2}$, early diastolic and late diastolic flow volumes were lower than those of the control group. In the ASO group, $\mathrm{ABI}, \mathrm{TcPO}_{2}$ and resistive index were lower than those of the control group.

To clarify the mechanism of reduced flow volume in the non-ASO group, we assigned the 60 patients of this group to three subgroups based on the levels of total flow volume of the popliteal artery. The characteristics of these groups are summarised in Table 3. The groups were similar in sex and age. Furthermore, 
Table 1. Characteristics of diabetic patients with (ASO group) or without (non-ASO group) occlusive arterial diseases in the lower extremities and age-matched non-diabetic subjects (control group)

\begin{tabular}{|c|c|c|c|}
\hline & Control group & Non-ASO group & ASO group \\
\hline Number & 16 & 60 & 16 \\
\hline Sex (male/female) & $8 / 8$ & $30 / 30$ & $14 / 2$ \\
\hline Age (years) & $59.8 \pm 7.1$ & $60.2 \pm 6.7$ & $62.1 \pm 4.9$ \\
\hline BMI $\left(\mathrm{kg} / \mathrm{m}^{2}\right)$ & $22.4 \pm 2.7$ & $23.1 \pm 3.9$ & $23.2 \pm 4.5$ \\
\hline Duration of diabetes (years) & - & $13.2 \pm 7.5$ & $17.4 \pm 10.0$ \\
\hline Treatment (D/OHD/I) & - & $5 / 27 / 28$ & $4 / 7 / 5$ \\
\hline FPG $(\mathrm{mmol} / \mathrm{l})$ & $5.22 \pm 0.42$ & $9.23 \pm 3.48^{c}$ & $8.66 \pm 2.18^{b}$ \\
\hline $\mathrm{HbA}_{1 \mathrm{c}}(\%)$ & $4.6 \pm 0.5$ & $8.8 \pm 1.7^{\mathrm{c}}$ & $8.6 \pm 1.3^{\mathrm{c}}$ \\
\hline Cholesterol $(\mathrm{mmol} / \mathrm{l})$ & $4.70 \pm 0.78$ & $5.09 \pm 1.00$ & $5.08 \pm 0.85$ \\
\hline HDL-cholesterol $(\mathrm{mmol} / \mathrm{l})$ & $1.19 \pm 0.40$ & $1.22 \pm 0.32$ & $1.17 \pm 0.38$ \\
\hline Triglycerides $(\mathrm{mmol} / \mathrm{l})$ & $1.11 \pm 0.22$ & $1.39 \pm 0.65$ & $1.47 \pm 0.53$ \\
\hline Systolic blood pressure $(\mathrm{mm} \mathrm{Hg})$ & $122 \pm 12$ & $131 \pm 18$ & $136 \pm 22$ \\
\hline Diastolic blood pressure $(\mathrm{mm} \mathrm{Hg})$ & $65 \pm 5$ & $70 \pm 10$ & $70 \pm 12$ \\
\hline Smokers $(\%)$ & $7(44 \%)$ & $19(32 \%)$ & $13(81 \%)^{\mathrm{d}}$ \\
\hline $\operatorname{MCV}(\mathrm{m} / \mathrm{sec})$ & $44.6 \pm 1.7$ & $39.0 \pm 6.8^{\mathrm{b}}$ & $39.4 \pm 4.2^{\mathrm{a}}$ \\
\hline $\operatorname{SCV}(\mathrm{m} / \mathrm{sec})$ & $44.3 \pm 1.7$ & $39.2 \pm 5.9^{\mathrm{b}}$ & $37.9 \pm 6.6^{\mathrm{b}}$ \\
\hline $\mathrm{CV}_{\mathrm{R}-\mathrm{R}}(\%)$ & $3.41 \pm 1.15$ & $2.14 \pm 1.29^{\mathrm{b}}$ & $2.28 \pm 0.93^{\mathrm{a}}$ \\
\hline Retinopathy (\%) & - & $41(68 \%)$ & $8(50 \%)$ \\
\hline Nephropathy (\%) & - & $20(33 \%)$ & $2(13 \%)$ \\
\hline
\end{tabular}

Data are expressed as means \pm SD. There were significant differences in $\mathrm{MCV}(p<0.01)$, $\mathrm{SCV}(p<0.01)$ and $\mathrm{CV}_{\mathrm{R}-\mathrm{R}}$ $(p<0.01)$ among the groups. ${ }^{\mathrm{a}} p<0.05,{ }^{\mathrm{b}} p<0.01,{ }^{\mathrm{c}} p<0.001$ vs the control group, ${ }^{\mathrm{d}} p<0.01$ vs the non-ASO group. D, diet; OHD, oral hypoglycaemic drugs; I, insulin
Table 2. Quantitative analyses of the peripheral circulation in diabetic patients with (ASO group) or without (non-ASO group) occlusive arterial diseases in the lower extremities and age-matched non-diabetic subjects (control group)

\begin{tabular}{lccc}
\hline & $\begin{array}{l}\text { Control } \\
\text { group }\end{array}$ & $\begin{array}{l}\text { Non-ASO } \\
\text { group }\end{array}$ & ASO group \\
\hline Number & 16 & 60 & 16 \\
$\mathrm{ABI}$ & $1.12 \pm 0.07$ & $1.15 \pm 0.13$ & $0.70 \pm 0.12^{\mathrm{c}, \mathrm{e}}$ \\
$\mathrm{TcPO}_{2}(\mathrm{~mm} \mathrm{Hg})$ & $68.9 \pm 7.6$ & $56.0 \pm 15.3^{\mathrm{b}}$ & $45.4 \pm 6.9^{\mathrm{c}, \mathrm{d}}$ \\
Heart rate (bpm) & $70 \pm 8$ & $73 \pm 10$ & $71 \pm 11$ \\
Flow volume & & & \\
(ml/min) & & & \\
$\quad$ Total & $88.9 \pm 21.9$ & $70.3 \pm 27.9$ & $79.0 \pm 30.9$ \\
$\quad$ Systolic & $80.2 \pm 16.8$ & $75.5 \pm 23.1$ & - \\
$\quad$ Early diastolic & $-7.3 \pm 6.2$ & $-12.5 \pm 8.6^{\mathrm{a}}$ & - \\
$\quad$ Late diastolic & $16.0 \pm 7.9$ & $7.2 \pm 9.0^{\mathrm{c}}$ & - \\
Resistive index & $0.96 \pm 0.03$ & $0.99 \pm 0.04$ & $0.85 \pm 0.12^{\mathrm{c}, \mathrm{e}}$ \\
\hline
\end{tabular}

Data are expressed as means \pm SD. There were significant differences in ABI $(p<0.001), \mathrm{TcPO}_{2}(p<0.001)$ and resistive index $(p<0.001)$ among the groups. ${ }^{\mathrm{a}} p<0.05,{ }^{\mathrm{b}} p<0.01$, ${ }^{\mathrm{c}} p<0.001$ vs the control group, ${ }^{\mathrm{d}} p<0.05,{ }^{\mathrm{e}} p<0.001$ vs the non-ASO group. Flow volumes of systolic, early diastolic and late diastolic cardiac phases were calculated from the integration of waveform. Resistive index is defined as (A-B)/A, where $\mathrm{A}$ is the systolic peak velocity and $\mathrm{B}$ is the end-diastolic velocity

there were no significant differences in duration of diabetes, FPG, $\mathrm{HbA}_{1 \mathrm{c}}$, cholesterol, HDL-cholesterol, triglycerides, sBP, dBP, frequency of smokers, $\mathrm{SCV}, \mathrm{CV}_{\mathrm{R}-\mathrm{R}}$ or frequency of retinopathy and nephropathy among the groups. A difference among the groups was found for MCV of the lowest group, which was lower than that of the highest group. The waveforms of the popliteal artery in these groups are shown in Figure 2. All non-ASO patients had a triphasic waveform. The lowest group (Fig.2) had, however, lower amplitudes of systolic and late diastolic components than those of the highest group (Fig.2). Magnetic resonance angiograms of the three groups, which reflected flow velocities greater than $5 \mathrm{~cm} / \mathrm{s}$, are shown in Figure 3. Foot vasculatures, such as dorsalis pedis, medial and lateral plantar arteries and plantar arch in one case of the highest group (Fig.3) were clearly visible. Vasculatures in the lowest group (Fig. 3) were, however, poorly shown indicating decreased flow velocities in the foot arteries. The quantitative analyses of the peripheral circulation in these groups are summarised in Table 4. There were significant differences in $\mathrm{TcPO}_{2}$, systolic flow volume, late diastolic flow volume and resistive index among the groups with the exception of ABI and early diastolic flow volume. In the lowest group, $\mathrm{TcPO}_{2}$, systolic and late diastolic flow volumes were lower and resistive index was higher than those of the highest group.

The PCr: $\mathrm{P}_{\mathrm{i}}$ ratio of resting plantar muscles in the ASO, non-ASO and control groups are shown in Table 5. There was a significant difference in the PCr: $P_{i}$ ratio among the groups, which was lower in the nonASO and ASO groups than in the control group. When the non-ASO group was categorised into three subgroups based on their levels of total flow volume of the popliteal artery, the lowest group had a reduction of the $\mathrm{PCr}$ content and a complementary increase in the $\mathrm{P}_{\mathrm{i}}$ content compared with those of the 

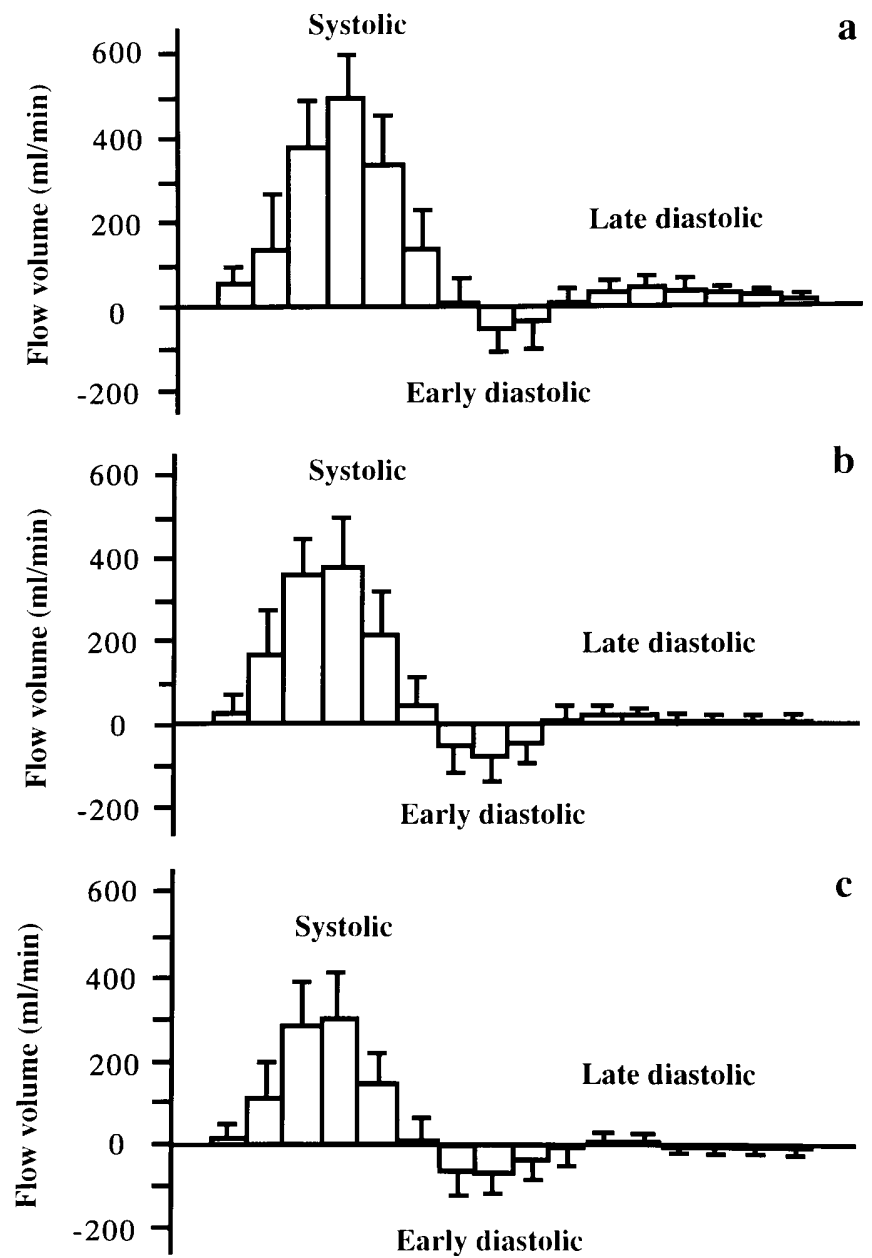

Fig. 2a-c. Waveform analyses of the popliteal artery in 60 diabetic patients without occlusive arterial diseases in the lower extremities. These patients were put into categories of the highest a, intermediate $\mathbf{b}$ and lowest $\mathbf{c}$ levels of total flow volume of the popliteal artery. Instantaneous flow volume at 16 equally spaced time points through the cardiac cycle were reconstructed. Data are expressed as means \pm SD

highest group. Consequently, there was a significant difference in the PCr: $P_{i}$ ratio among the groups, which was lower in the lowest group than in the highest group (Table 5).

To characterise clinical variables for determining the PCr: $P_{i}$ ratio of plantar muscles in the non-ASO group, we did stepwise multiple regression analysis of eight vascular factors $\left(\mathrm{ABI}, \mathrm{TcPO}_{2}\right.$, heart rate, total, systolic, early diastolic and late diastolic flow volumes and resistive index), three neurological factors (MCV, SCV and $\mathrm{CV}_{\mathrm{R}-\mathrm{R}}$ ) and two factors of diabetic microangiopathy (retinopathy and nephropathy). Late diastolic flow volume $(\beta$ value $=0.07, F$ value $=6.37), \operatorname{MCV}(\beta$ value $=0.16, F$ value $=17.13)$ and nephropathy $(\beta$ value $=-0.90, F$ value $=9.00)$ were identified as significant independent variables for determining the PCr: $\mathrm{P}_{\mathrm{i}}$ ratio $\left(r^{2}=0.484\right.$, $p<0.001)$. In addition, simple linear regression anal- ysis between late diastolic flow volume and resistive index showed a negative correlation $(r=-0.72$, $p<0.001)$.

\section{Discussion}

We have recently shown that diabetic patients with neuropathic foot ulcers have lower phosphocreatine content in their plantar muscles than non-ulcerated patients, although they had no clinical signs of occlusive arterial diseases in the lower extremities assessed by ankle brachial index, transcutaneous $\mathrm{O}_{2}$ tension on the dorsal foot and total flow volume of the popliteal artery [6]. The ulcerated patients were also characterised by diffused fatty infiltration and increased intracellular $\mathrm{pH}$ of plantar muscles, indicating that these abnormalities are not related to a mechanism that causes ischaemia. Furthermore, we also identified motor nerve function and severity of nephropathy as significant independent factors for determining these abnormalities of plantar muscles in ulcerated patients [6]. In the present study, we further analysed the abnormal peripheral circulation caused by either occlusive arterial diseases or increased arterial resistance in the lower extremities using gated magnetic resonance two-dimensional cine-mode phase-contrast (2D-cine-PC) imaging and flow analysis of the popliteal artery.

Peripheral vascular disease is believed, in part, to cause diabetic peripheral neuropathy. Peripheral nerve function is inversely correlated to transcutaneous oxygenation in diabetic patients with occlusive arterial diseases in the lower extremities [13]. Restoration of arterial blood supply and tissue oxygenation following reconstructive surgery is therefore accompanied by an improvement of peripheral nerve function [13]. Two-dimensional cine-mode phase-contrast imaging provides useful flow data in both normal and diseased arteries. The non-diabetic subjects (control group) showed that the systolic, early diastolic and late diastolic phases of the cardiac cycle produce three distinct segments in the popliteal artery with a typical triphasic waveform. Diabetic patients with occlusive arterial lesions and developing collateral arterial branches from the aortic bifurcation to the femoral artery (ASO group) had, however, an abnormal monophasic waveform. The physical and flowmechanical reasons for the generation of this flow phenomenon in peripheral artery were considered as follows. Firstly the triphasic waveform is dampened in the diseased and collateral arteries, reducing the amplitude and broadening the forward flow with the replacement of early diastolic flow reversal by persistent forward flow during the cardiac cycle when a haemodynamically relevant arterial obstruction occurs [7]. Secondly, the degree of early diastolic flow reversal depends on the resistance of distal artery 

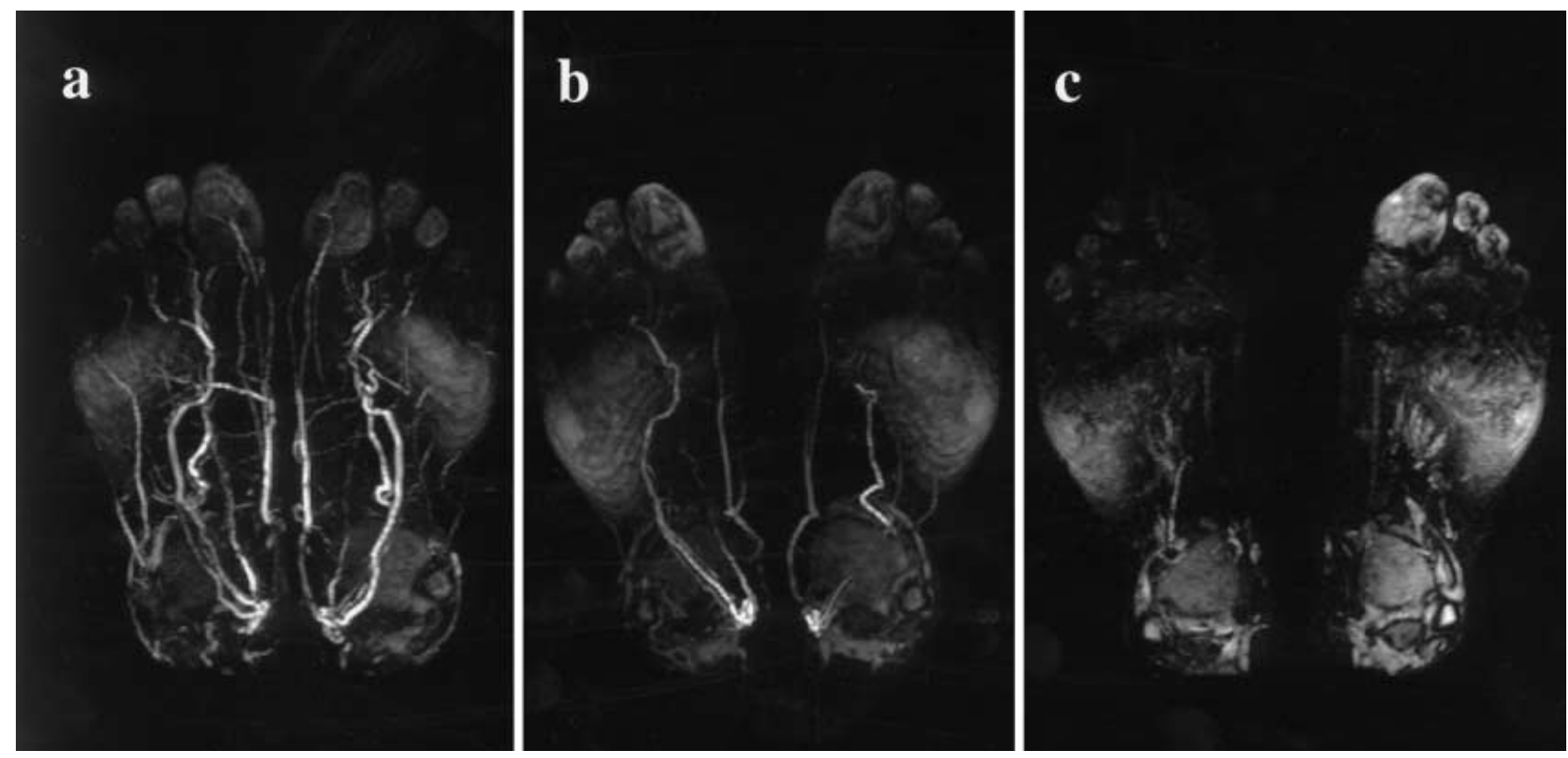

Fig. 3a-c. Three cases of foot vasculatures using magnetic resonance angiography with no contrast medium in each of the highest a, intermediate b and lowest c groups. We placed 60 diabetic patients without occlusive arterial diseases in the lower extremities into three subgroups based on the levels of total flow volume of the popliteal artery

[3]. In our study, the ASO group had a lower resistive index of popliteal artery than that of the control group. Thus, we could not see an early diastolic retrograde flow in this group.
The accuracy and reproducibility of 2D-cine-PC imaging to measure flow volume for both triphasic and monophasic waveforms created from a pulsatile pump has been reported [14]. Furthermore, Doppler sonographic evaluation is most analogous to $2 \mathrm{D}$ cine-PC imaging, insofar as both methods measure the vector component of flow along an axis. When the feasibility of 2D-cine-PC imaging was compared with colour-coded Doppler sonography, the velocity waveforms acquired using both methods correlated well [7]. The present examination time was similar to that of Doppler sonography. Some characteristics of

Table 3. Characteristics of diabetic patients without occlusive arterial diseases in the lower extremities classified into three subgroups based on the levels of total flow volume of popliteal artery

\begin{tabular}{llll}
\hline & Highest group & Intermediate group & Lowest group \\
& $101.6 \pm 15.0 \mathrm{ml} / \mathrm{min}$ & $68.3 \pm 7.1 \mathrm{ml} / \mathrm{min}$ & $20.9 \pm 14.1 \mathrm{ml} / \mathrm{min}$ \\
\hline Number & 20 & 20 & $10 / 10$ \\
Sex (male/female) & $10 / 10$ & $10 / 10$ & $60.8 \pm 6.9$ \\
Age (years) & $58.8 \pm 6.3$ & $61.2 \pm 6.9$ & $21.6 \pm 2.5$ \\
BMI (kg/m ${ }^{2}$ ) & $23.7 \pm 2.6$ & $23.3 \pm 3.8$ & $11.8 \pm 6.3$ \\
Duration of diabetes (years) & $13.3 \pm 8.7$ & $14.5 \pm 7.4$ & $2 / 9 / 9$ \\
Treatment (D/OHD/I) & $0 / 10 / 10$ & $3 / 8 / 9$ & $10.13 \pm 4.61$ \\
FPG (mmol/l) & $9.12 \pm 2.16$ & $8.45 \pm 3.21$ & $8.83 \pm 1.84$ \\
HbA $(\%)$ & $8.85 \pm 1.45$ & $8.65 \pm 1.93$ & $4.96 \pm 0.80$ \\
Cholesterol (mmol/l) & $4.97 \pm 1.09$ & $5.61 \pm 0.69$ & $1.27 \pm 0.34$ \\
HDL-cholesterol (mmol/l) & $1.09 \pm 0.31$ & $1.31 \pm 0.29$ & $1.34 \pm 0.50$ \\
Triglycerides (mmol/l) & $1.66 \pm 0.64$ & $1.39 \pm 0.64$ & $128 \pm 17$ \\
Systolic blood pressure (mm Hg) & $133 \pm 21$ & $133 \pm 16$ & $70 \pm 9$ \\
Diastolic blood pressure (mm Hg) & $70 \pm 13$ & $69 \pm 9$ & $7(35 \%)$ \\
Smokers (\%) & $9(45 \%)$ & $3(15 \%)$ & $35.5 \pm 6.6^{\mathrm{a}}$ \\
MCV (m/sec) & $41.2 \pm 5.5$ & $40.2 \pm 7.0$ & $37.3 \pm 5.6$ \\
SCV (m/sec) & $40.7 \pm 6.3$ & $39.8 \pm 5.5$ & $2.45 \pm 1.35$ \\
CV R-R $\%$ ) & $2.05 \pm 1.18$ & $1.93 \pm 1.33$ & $15(75 \%)$ \\
Retinopathy (\%) & $14(70 \%)$ & $12(60 \%)$ & $6(30 \%)$ \\
Nephropathy (\%) & $8(40 \%)$ & $6(30 \%)$ & \\
\hline Data are expressed &
\end{tabular}

Data are expressed as means \pm SD. There was a significant difference in MCV $(p<0.05)$ among the groups. ${ }^{a} p<0.05$ vs the highest group. D, diet; OHD, oral hypoglycaemic drugs; I, insulin 
Table 4. Quantitative analyses of peripheral circulation in diabetic patients without occlusive arterial diseases in the lower extremities classified into three subgroups based on the levels of total flow volume of popliteal artery

\begin{tabular}{|c|c|c|c|}
\hline & $\begin{array}{l}\text { Highest group } \\
101.6 \pm 15.0 \mathrm{ml} / \mathrm{min}\end{array}$ & $\begin{array}{l}\text { Intermediate group } \\
68.3 \pm 7.1 \mathrm{ml} / \mathrm{min}\end{array}$ & $\begin{array}{l}\text { Lowest group } \\
40.9 \pm 14.1 \mathrm{ml} / \mathrm{min}\end{array}$ \\
\hline Number & 20 & 20 & 20 \\
\hline $\mathrm{ABI}$ & $1.18 \pm 0.16$ & $1.16 \pm 0.11$ & $1.10 \pm 0.09$ \\
\hline $\mathrm{TcPO}_{2}(\mathrm{~mm} \mathrm{Hg})$ & $68.6 \pm 11.1$ & $58.1 \pm 11.8^{\mathrm{a}}$ & $47.1 \pm 15.7^{\mathrm{c}, \mathrm{d}}$ \\
\hline Heart rate (bpm) & $75 \pm 12$ & $74 \pm 7$ & $72 \pm 11$ \\
\hline \multicolumn{4}{|c|}{ Flow volume (ml/min) } \\
\hline Systolic & $96.3 \pm 18.1$ & $75.5 \pm 12.7^{\mathrm{c}}$ & $54.6 \pm 16.0^{\mathrm{c}, \mathrm{e}}$ \\
\hline Early diastolic & $-9.6 \pm 8.5$ & $-13.5 \pm 9.3$ & $-14.4 \pm 7.6$ \\
\hline Late diastolic & $14.9 \pm 7.9$ & $6.3 \pm 6.8^{b}$ & $0.6 \pm 5.8^{\mathrm{c}, \mathrm{d}}$ \\
\hline Resistive index & $0.97 \pm 0.03$ & $0.99 \pm 0.04^{\mathrm{a}}$ & $1.02 \pm 0.03^{c}$ \\
\hline
\end{tabular}

Data are expressed as means $\pm \mathrm{SD}$. There were significant differences in $\mathrm{TcPO}_{2} \quad(p<0.001)$, systolic flow volume $(p<0.001)$, late diastolic flow volume $(p<0.001)$ and resistive index $(p<0.001)$ among the groups. ${ }^{\mathrm{a}} p<0.05,{ }^{\mathrm{b}} p<0.01$, ${ }^{\mathrm{c}} p<0.001$ vs the highest group, ${ }^{\mathrm{d}} p<0.05,{ }^{\mathrm{e}} p<0.001$ vs the in-

Table 5. ${ }^{31} \mathrm{P}$-magnetic resonance spectroscopic analyses of plantar muscles in diabetic patients with (ASO group) or without (non-ASO group) occlusive arterial diseases in the lower extremities and age-matched non-diabetic subjects (control group)

\begin{tabular}{lll}
\hline Total subjects & Number & PCr: Pi ratio \\
\hline Control group & 16 & $6.86 \pm 0.85$ \\
Non-ASO group & 60 & $4.76 \pm 2.40^{\mathrm{b}}$ \\
ASO group & 16 & $5.07 \pm 0.84^{\mathrm{a}}$ \\
\hline Non-ASO group & Number & PCr: Pi ratio \\
\hline Highest group & 20 & $5.69 \pm 2.46$ \\
Intermediate group & 20 & $4.91 \pm 2.52$ \\
Lowest group & 20 & $3.71 \pm 1.87^{\mathrm{c}}$ \\
\hline
\end{tabular}

Data are expressed as means $\pm \mathrm{SD}$. The non-ASO group is classified into three subgroups based on the levels of total flow volume of popliteal artery. There were significant differences in $\mathrm{PCr}: \mathrm{P}_{i}$ ratio among the groups in total subjects $(p<0.01)$ and subgroups in the non-ASO group $(p<0.05)$. ${ }^{\mathrm{a}} p<0.05,{ }^{\mathrm{b}} p<0.01$ vs the control group, ${ }^{\mathrm{c}} p<0.05$ vs the highest group

2D-cine-PC imaging could, however, be superior to those of Doppler sonography. Firstly high frequency at $63.9 \mathrm{MHz}$ provided excellent resolution of deep vasculatures through thick body tissues or calcified plaque of the vessel. Secondly, the large size of quadrature coil with $20 \mathrm{~cm}$ field of view simultaneously covers both sides of the popliteal arteries as well as different locations along the same vessel. Thirdly, the angle of view is perpendicular to the flow direction with no operator dependency in selection of angle correction between the transducer axis and the vessel.

We analysed the waveform and total flow volume of the popliteal artery in diabetic patients who showed no clinical signs and symptoms of occlusive arterial diseases in the lower extremities (non-ASO termediate group. Flow volumes of systolic, early diastolic and late diastolic cardiac phases were calculated from the integration of waveform. Resistive index is defined as (A-B)/A, where $\mathrm{A}$ is the systolic peak velocity and $\mathrm{B}$ is the end-diastolic velocity

group). Some diabetic patients in this group suffered from reduced total flow volume and had an abnormal triphasic waveform with decreased amplitudes of both systolic and late diastolic phases. These patients had a higher resistive index, which was associated with increased vascular resistance [15] and decreased vascular compliance [16]. Furthermore, magnetic resonance angiograms in these patients showed lower flow velocities in foot arteries. These findings indicate that diabetes can lead to high arterial wall stiffness $[17,18]$ and resistance to blood flow [19].

Some diabetic patients in the non-ASO group suffered from reduced energy metabolism of plantar muscles. In the present statistical analysis, late diastolic flow volume of the popliteal artery in addition to the previously reported motor nerve function and severity of nephropathy were identified as statistically independent risk factors for determining these abnormalities [6]. As a consequence of the negative correlation between late diastolic flow volume and the resistive index of the popliteal artery, this vascular factor is associated with arterial resistance to blood flow in the lower extremities. Furthermore, this vascular factor was a much lower amplitude than that of the systolic component in waveform and was more easily affected by arterial wall stiffness than resistive index. The presence of medial arterial calcification in the foot has been reported to be more frequent in neuropathic diabetic patients with a history of foot ulceration [20]. Thus, increased vascular resistance could be biologically linked to neurological abnormalities. In diabetic nephropathy, its pathogenesis is known to be a consequence of microangiopathy [21] and intrarenal arteriosclerosis [22]. Carotid artery stiffness assessed by sonography is associated with the development of microalbuminuria [23]. Arterial resistance of the lower extremities in our study was not, however, associated with the severity of nephropathy. 
Waveform analysis of the popliteal artery using gated magnetic resonance 2D-cine-PC imaging is a powerful tool that can define impaired peripheral circulation in the lower limbs. Occlusive arterial lesions with developing collateral arteries from the aortic bifurcation to the femoral artery is characterised by an abnormal monophasic waveform and results in low perfusion pressure in the lower limbs. Diabetic patients who have increased arterial resistance to blood flow show an abnormal triphasic waveform with lower amplitudes of systolic and late diastolic components resulting in inadequate blood supply to the lower limbs. This impaired peripheral circulation of the lower limbs caused by either occlusive arterial disease or increased arterial resistance in diabetic patients could be a high-risk for foot lesions.

Acknowledgements. This study was supported in part by a Grant-in-Aid for the Development of New Clinical Technology, and Grants-in-Aid for Scientific Research (A) (No. 10358017) and International Scientific Research (No. 09044287) from the Ministry of Education, Science, Sports and Culture, Japan.

\section{References}

1. Sosenko JM, Kato M, Soto R, Bild DE (1990) Comparison of quantitative sensory-threshold measures for their association with foot ulceration in diabetic patients. Diabetes Care 13: 1057-1061

2. Shaw JE, van Schie CH, Carrington AL, Abbott CA, Boulton AJ (1998) An analysis of dynamic forces transmitted through the foot in diabetic neuropathy. Diabetes Care 21: 1955-1959

3. Polak JF (1993) Arterial sonography: efficacy for the diagnosis of arterial disease of the lower extremity. Am J Roentgenol 161: 235-243

4. Orchard TJ, Strandness DE (1993) Assessment of peripheral vascular disease in diabetes. Diabetes Care 16: 1199-1209

5. Pecoraro RE, Ahroni JH, Boyko EJ, Stensel VL (1991) Chronology and determinants of tissue repair in diabetic lower-extremity ulcers. Diabetes 40: 1305-1313

6. Suzuki E, Kashiwagi A, Hidaka $\mathrm{H}$ et al. (2000) ${ }^{1} \mathrm{H}-$ and ${ }^{31} \mathrm{P}-$ magnetic resonance spectroscopy and imaging as a new diagnostic tool to evaluate neuropathic foot ulcers in Type II diabetic patients. Diabetologia 43: 165-172

7. Caputo GR, Masui T, Gooding GA, Chang JM, Higgins CB (1992) Popliteal and tibioperoneal arteries: feasibility of two-dimensional time-of-flight MR angiography and phase velocity mapping. Radiology 182: 387-392
8. World Health Organization Study Group on Diabetes Mellitus (1985) Technical report series No 727, WHO, Geneva

9. Diabetic Retinopathy Study Research Group (1981) Report VII: a modification of the Airlie House classification of diabetic retinopathy. Invest Ophthalmol Vis Sci 21: 210-226

10. Nelson TR, Pretorius DH (1988) The Doppler signal: where does it come from and what does it mean ? Am J Roentgenol 151: 439-447

11. Bottomley PA, Foster TB, Darrow RD (1984) Depth-resolved surface-coil spectroscopy (DRESS) for in vivo ${ }^{1} \mathrm{H}$, ${ }^{31} \mathrm{P}$, and ${ }^{13} \mathrm{C}$ NMR. J Magn Reson 59: 338-342

12. Taylor DJ, Bore PJ, Styles P, Gadian DG, Radda GK (1983) Bioenergetics of intact human muscle. $\mathrm{A}^{31} \mathrm{P}$ nuclear magnetic resonance study. Mol Biol Med 1: 77-94

13. Young MJ, Veves A, Walker MG, Boulton AJ (1992) Correlations between nerve function and tissue oxygenation in diabetic patients: further clues to the aetiology of diabetic neuropathy ? Diabetologia 35: 1146-1150

14. McCauley TR, Pena CS, Holland CK, Price TB, Gore JC (1995) Validation of volume flow measurements with cine phase-contrast MR imaging for peripheral arterial waveforms. J Magn Reson Imaging 5: 663-668

15. Halpern EJ, Merton DA, Forsberg F(1998) Effect of distal resistance on Doppler US flow patterns. Radiology 206: 761-766

16. Bude RO, Rubin JM (1999) Relationship between the resistive index and vascular compliance and resistance. Radiology 211: 411-417

17. Yamasaki Y, Kawamori R, Matsushima H et al. (1995) Asymptomatic hyperglycaemia is associated with increased intimal plus medial thickness of the carotid artery. Diabetologia 38: 585-591

18. Sims TJ, Rasmussen LM, Oxlund H, Bailey AJ (1996) The role of glycation cross-links in diabetic vascular stiffening. Diabetologia 39: 946-951

19. Emoto M, Nishizawa Y, Kawagishi T et al. (1998) Stiffness indexes $\beta$ of the common carotid and femoral arteries are associated with insulin resistance in NIDDM. Diabetes Care 21: 1178-1182

20. Young MJ, Adams JE, Anderson GF, Boulton AJ, Cavanagh PR (1993) Medial arterial calcification in the feet of diabetic patients and matched non-diabetic control subjects. Diabetologia 36: 615-621

21. Raine AE, Bilous RW (1996) End-stage renal disease in NIDDM: a consequence of microangiopathy alone? Diabetologia 39: 1673-1675

22. Boeri D, Derchi LE, Martinoli C et al. (1998) Intrarenal arteriosclerosis and impairment of kidney function in NIDDM subjects. Diabetologia 41: 121-124

23. Lambert J, Smulders RA, Aarsen M, Donker AJ, Stehouwer CDA (1998) Carotid artery stiffness is increased in microalbuminuric IDDM patients. Diabetes Care 21: 99-103 